\title{
Silicon Corrole based paper strips for the visual determination of fluoride ion
}

\author{
G. Pomarico ${ }^{1}$, D. Monti ${ }^{1}$, C. Di Natale ${ }^{2}$, R. Paolesse ${ }^{1}$ \\ ${ }^{1}$ Dipartimento di Scienze e Tecnologie Chimiche ${ }^{2}$ Dipartimento di Ingegneria Elettronica, \\ University of Rome Tor Vergata, Via della Ricerca Scientifica 1, 00133 Rome, Italy, \\ roberto.paolesse@uniroma2.it
}

\begin{abstract}
:
A Silicon corrole complex has been utilised as a novel sensing material for the development of optode for fluoride ion detection. The fluoride binding induced UV-visible spectral changes exploited for the anion detection. The corrole complex was deposited onto paper strips from THF solution. The corrole spots changed from red pink to green color upon fluoride exposure. The color changes can be visually appreciated down to a fluoride ion concentration of $10^{-5} \mathrm{~mol} / \mathrm{L}$.
\end{abstract}

Key words: paper sensors, porphyrinoids, fluoride, optical sensors, optode.

\section{Introduction}

The development of simple systems for the control of drinkable water is nowadays an urgent task. Among the different analytes to be controlled, fluoride ion is one of the most important, because its content should be maintained in a defined window: fluoride is in fact essential for dental and skeletal care, but in high levels it is toxic and can lead to chronic neurodegenerative diseases [1]. The need of simple system able to monitor the fluoride content of water supplies is for this reason highly recommended. However the development of sensitive and selective sensors for fluoride ion detection is still a challenging task; among others, potentiometric ion selective electrodes have been reported for fluoride analysis, using metalloporphyrins as ionophores, although some sensitivity and selectivity problems have limited their success [2]. Metalloporphyrin based optical devices seem to be a promising alternative for the development of simple systems able to detect fluoride ions, although the sensitivity and selectivity also in this case limited the sensor performances [2]. From this point of view, we have recently pointed out the preparation of Silicon complexes of corrole, a contracted porphyrinoid characterized by brilliant optical features. Taking advantage of the UV-visible spectral changes induced by fluoride ion coordination on the UV-visible spectrum of $\mathrm{Si}$ corrole, we report in this work the development of simple, visual test papers for fluoride ion determination in drinkable water samples.

\section{Experimental}

Si corrole (Figure 1) was synthesised and tested in our laboratories according to the common methods. The PVC/DOS (PolyVynylchloride:bis-ethylhexyl sebacate = $1: 2$ by weight) membranes containing $1 \mathrm{wt} \%$ of Si corrole, $10-20 \mathrm{~mol} \%$ (relative to ionophore) of cationic lipophilic additive tetradodecylammonium chloride were tested.

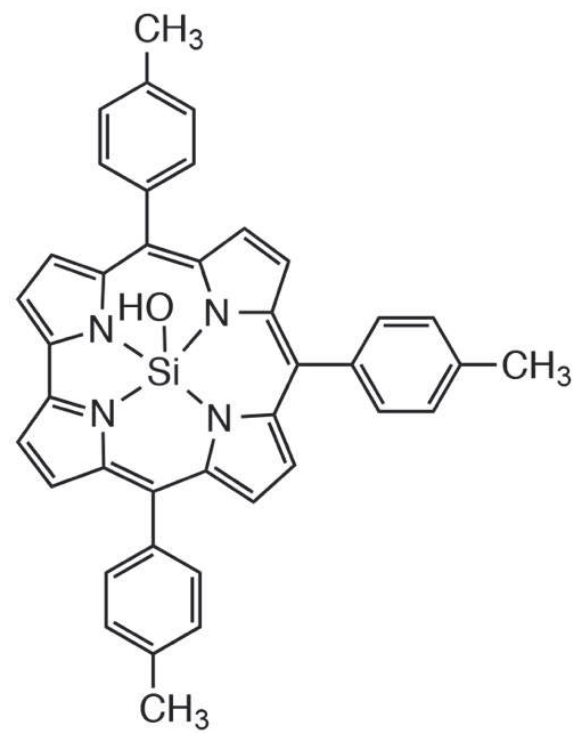

Figure 1. Molecular structure of Si corrole 
Membranes were deposited on modified glass slide transducers. The optical responses of $\mathrm{Si}$ corrole membranes towards several anions were examined. Fluoride ion calibrations were performed by adjusting the calculated amounts of concentrated stock solutions varying the concentration of ions stepwise from $10^{-7}$ to $10^{-2}$ $\mathrm{M}$ at every $100 \mathrm{~s}$. To ensure no $\mathrm{pH}$ interferences with measurements, solutions were buffered with MES (10 mM, pH 5.5). To test the potentialities of the developed paper strips for the analysis of real sample, we have studied the fluoride content in a commercial toothpaste.

\section{Results and Discussion}

The large affinity of Silicon cation toward the Fluoride anion triggered our interest about the exploitation of this metallo-corrolate for the $\mathrm{F}^{-}$ detection. We started to investigated the affinity of $\mathrm{Si}$ corrole toward $\mathrm{F}^{-}$in organic solvents with a different polarity. A $10^{-6} \mathrm{M}$ solution of 1 in $\mathrm{CH}_{2} \mathrm{Cl}_{2}$ or DMSO was treated with an increasing amount of a $1 \mathrm{M}$ standard solution of Bu${ }_{4} \mathrm{NF}$ in THF. A variation of the UVvis spectrum was observed: the main peak at $413 \mathrm{~nm}$ disappeared, replaced by a split Soret band at 424 and $436 \mathrm{~nm}$, while in the $Q$ region, the main absorbance was red shifted to $630 \mathrm{~nm}$ (Figure 2). These spectral changes were peculiar of fluoride binding, because no changes were observed by addition of a series of different anions, such as $\mathrm{Cl}^{-}, \mathrm{Br}^{-}, \mathrm{I}^{-}, \mathrm{CH}_{3} \mathrm{CO}_{2}^{-}$, $\mathrm{NO}_{2}^{-}, \mathrm{H}_{2} \mathrm{PO}_{4}^{-}$, confirming the $\mathrm{Si}$ corrole selectivity in fluoride binding.

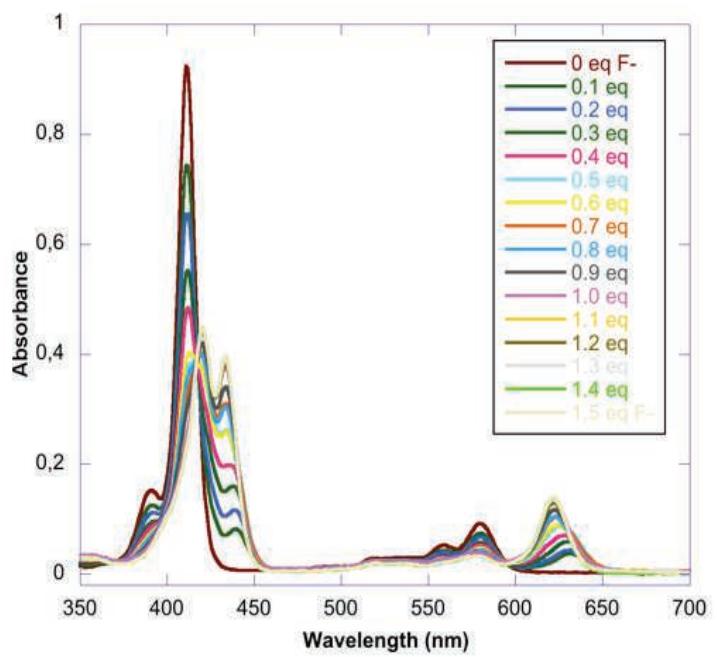

Fig. 2. UV-visible spectral changes of Si corrole upon fluoride ion addition.

To explore the ability of this corrole complex to develop simple optode for $\mathrm{F}^{-}$detection, we deposited the corrole complex onto Whatman Grade 42 ashless filter paper strips from THF solutions. Paper strips (PSs) can in fact permit to develop easy to use, inexpensive and disposable optodes, user-friendly for visual detection of the target analyte.

The obtained PSs were immersed in solutions containing different concentrations of fluoride ion. The red-pink to green color change can be naked eye observed down to fluoride concentration of $10^{-5} \mathrm{~mol} / \mathrm{L}$, which is lower than the limit fixed by WHO for drinkable water [3].

To explore the potentialities of the developed optodes for the determination of fluoride in real samples, we exploited the developed PPs for the determination of the fluoride ion content in a commercial toothpaste. The PP was immersed into the water solution obtained by extraction of the toothpaste and a rapid color change was observed, indicating the detection of fluoride ion (figure 3)

The obtained result shows the utility of the developed POCT system for MC-LR detection in drinking water on the levels of WHO provisional guideline value $(1 \mu \mathrm{g} / \mathrm{L})$, with no need of laboratory equipment, no sample pretreatment and possibility to perform in-field analysis in combination with familiar devices for signal acquisition.

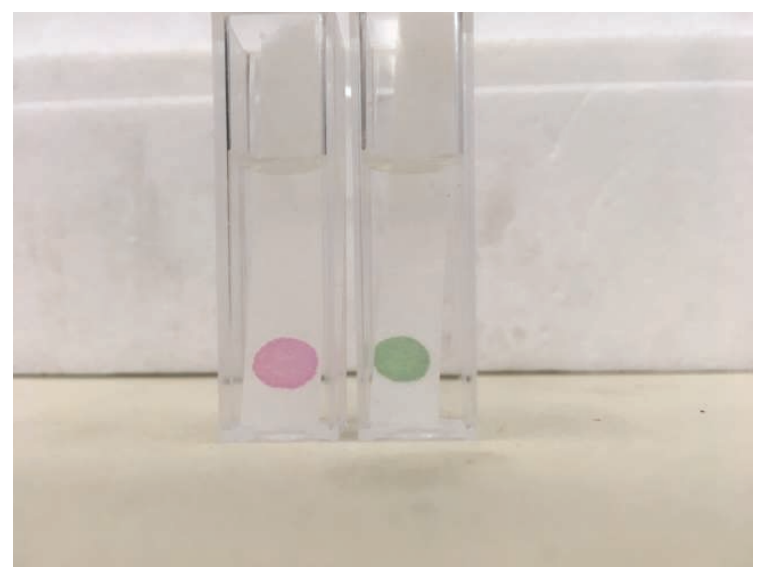

Fig. 3. Paper strip color change upon exposure to fluoride ion contained in commercial toothpaste.

\section{References}

[1] D. L. Ozsvath, Fluoride and environmental health: a review. Rev. Environ. Sci. Biotechnol. 8 (1), 5979 (2009); doi: 10.1007/s11157-008-9136-9

[2] R. Paolesse, S. Nardis, D. Monti, M. Stefanelli, C. Di Natale, Porphyrinoids for Chemical Sensor Applications. Chem. Rev., 117, 2517-2583 (2017); doi: 10.1021/acs.chemrev.6b00361.

[3] World Health Organization, 2011. Guidelines for Drinking-water Quality. fourth ed. ISBN: 978924 154815. 